\title{
Diversification of the plant-specific hybrid glycine-rich protein (HyGRP) genes in cereals
}

\author{
Kenji Fujino*, Mari Obara and Koji Sato \\ NARO Hokkaido Agricultural Research Center, National Agricultural Research Organization, Sapporo, Japan
}

\section{Edited by:}

Michael R. Schlappi, Marquette

University, USA

Reviewed by:

Rita Sharma, Jawaharlal Nehru

University, India

Jing Ning, Michigan State

University, USA

Dominique Job, Centre National de

la Recherche Scientifique, France

*Correspondence:

Kenji Fujino, NARO Hokkaido

Agricultural Research Center,

National Agricultural Research

Organization, Hitsujigaoka 1,

Sapporo 062-8555, Japan

e-mail:kfujino@affrc.go.jp
Plant-specific hybrid proline- or glycine-rich proteins (HyP/GRPs) are involved in diverse gene functions including plant development and responses to biotic and abiotic stresses. The quantitative trait locus, qLTG3-1, enhances seed germination in rice under low-temperature conditions and encodes a member with a glycine-rich motif of the HyP/GRP family. The function of this gene may be related to the weakening of tissue covering the embryo during seed germination. In the present study, the diversification of the HyP/GRP gene family was elucidated in rice based on phylogenetic relationships and gene expression levels. At least 21 members of the HyP/GRP family have been identified in the rice genome and clustered in five regions on four chromosomes by tandem and chromosomal duplications. Of these, OsHyPRP05 (qLTG3-1) and its paralogous gene, OsHyPRP21, had a glycine-rich motif. Furthermore, orthologous genes with a glycine-rich motif and the HyP/GRP gene family were detected in four genome-sequenced monocots: 12 in barley, 10 in Brachypodium, 20 in maize, and 28 in sorghum, using a BLAST search of qLTG3-1 as the query. All members of the HyP/GRP family in these five species were classified into seven main groups, which were clustered together in these species. These results suggested that the HyP/GRP gene family was formed in the ancestral genome before the divergence of these species. The collinearity of chromosomal regions around qLTG3-1 and its orthologous genes were conserved among rice, Brachypodium, sorghum, and maize, indicating that $q L T G 3-1$ and orthologous genes conserve gene function during seed germination.

Keywords: gene family, HyP/GRPs, orthologue, Oryza sativa L., rice

\section{INTRODUCTION}

Gene duplication contributes to genetic complexity during evolution (Hughes, 1994; Lynch and Force, 2000; Gu et al., 2003). One of the duplicated genes becomes a pseudogene through the accumulation of deleterious mutations, or duplicated genes adopt a subset of functions from the ancestral gene. Extant plant genomes may all result from whole genome duplication and diploidization (Adams and Wendel, 2005). Major losses, structural and functional divergence, or concerted evolution have been observed in eukaryote genomes via their whole genome duplication (Ahn and Tanksley, 1993; Wang et al., 2005; Scannell et al., 2006; Sjödin et al., 2008). The availability of the genome sequences of major cereals, rice (International Rice Genome Sequencing Project, 2005), sorghum (Paterson et al., 2009), barley (The International Barley Genome Sequencing Consortium, 2012), and maize (Schnable et al., 2009), has provided an opportunity for whole genome annotations and comparative genomic research to understand functional diversity among gene families.

Hybrid proline- or glycine-rich proteins (HyP/GRP) are plantspecific and putative cell-wall/plasma membrane-associated proteins. These mature proteins have two distinct domains: a hydrophilic proline-rich or glycine-rich repetitive domain (PRD or GRD, respectively) at the N-terminus, and a hydrophobic domain with eight cysteine residues in a specific order called the eight-cysteine motif (8CM) at the C-terminus. Although the HyP/GRP family has a unique structural feature, their proposed molecular functions vary and include plant development (Wu et al., 1993; Holk et al., 2002; Blanco-Portales et al., 2004), responses to various stresses including cold/heat, drought, and salinity (Deutch and Winicov, 1995; Goodwin et al., 1996; Zhang and Schläppi, 2007; Fujino et al., 2008b; Priyanka et al., 2010; Tan et al., 2013), and defenses against pathogens (Josè-Estanyol et al., 1992; He et al., 2002; Bouton et al., 2005; Weyman et al., 2006; Jung et al., 2009; Yeom et al., 2012). HyP/GRPs form a multi-gene family in plant species and have different gene expression profiles (Dvoráková et al., 2007). Therefore, little is known about their molecular functions and diversifications in plant development and responses to biotic and abiotic stresses.

Rice, as one of the most important cereals in the world, has become the most important model cereal for functional genomics. In many Asian countries, the direct seeding method has become increasingly important (Dingkuhn et al., 1992). Therefore, low temperature during the sowing at high altitudes and latitudes delays emergence of the rice seedling from water (Peterson et al., 1978), causing serious decreases of yields. We previously identified a quantitative trait locus (QTL) for low temperature tolerance at the seed germination stage, qLTG3-1 (Fujino et al., 2004, 2008b). This gene encodes one member with 
a glycine-rich motif of the HyP/GRP gene family in the rice genome. Although the molecular function of qLTG3-1 remains unknown, histological analyses indicated that the tissue-specific expression of $q L T G 3-1$ is closely associated with the vacuolation of cells in the tissues covering the embryo. Based on these findings, qLTG3-1 was considered to be involved in tissue weakening. Genome-wide expression analysis demonstrated that genes involved in defense responses were up-regulated by qLTG3-1 (Fujino and Matsuda, 2010). These findings indicated that the expression of $q L T G 3-1$ was necessary for the expression of defense response genes in low-temperature germinability in rice.

Genes that are highly similar to qLTG3-1 have been shown to exist in the genomes of plants other than rice (Fujino et al., 2008 b). Thus, it remains unclear whether these genes have the same function as qLTG3-1. In the present study, the phylogenetic relationships between and gene expression profiles of HyP/GRPs were characterized in rice to clarify whether other members had redundant or novel functions. Genome sequencing enabled us to perform comprehensive surveys of orthologous gene families across species (Hamilton and Buell, 2012). Comparative genomic analysis of the chromosomal regions around the qLTG3-1 orthologous genes in monocots including rice (International Rice Genome Sequencing Project, 2005), sorghum (Paterson et al., 2009), barley (The International Barley Genome Sequencing Consortium, 2012), maize (Schnable et al., 2009), and Brachypodium (The International Brachypodium Initiative, 2010) strongly suggested that qLTG3-1 orthologous genes with a glycine-rich motif have conserved gene function.

\section{MATERIALS AND METHODS PLANT MATERIALS}

The rice varieties Hokkaiwase, Kitaake, and Hoshinoyume were used for the gene expression analysis. The genotypes of qLTG3-1 in Hokkaiwase, Kitaake, and Hoshinoyume were the wild type, single amino acid substitute type, and loss-of-function type, respectively (Fujino and Iwata, 2011; Fujino and Sekiguchi, 2011). These varieties were cultivated in an experimental paddy field at Hokkaido Agricultural Research Center, Sapporo, Japan, $43^{\circ} 00^{\prime} \mathrm{N}$ latitude, in 2013. Seeds were harvested at the maturing stage and were then maintained at room temperature. These seeds were used in experiments 4 months after harvesting. Seeds were incubated at 15 and $30^{\circ} \mathrm{C}$ in dark conditions (Fujino et al., 2004). After being incubated, seed samples from each time point were frozen immediately in liquid nitrogen and stored at $-80^{\circ} \mathrm{C}$ for RNA extraction.

In the analysis of the sequence diversity of Os10g0554800, a paralogous gene of $q L T G 3-1$, a set of 58 genetically diverse varieties was used, which represents the wide genetic diversity among cultivated rice varieties termed world rice core collection (WRC) (Kojima et al., 2005). Seeds were provided by the Local Independent Administrative Agency Hokkaido Research Organization and National Institute of Agrobiological Sciences, Japan.

\section{DATABASE SEARCH FOR HyP/GRP GENES}

To identify the HyP/GRP gene family in the rice genome, we employed BlastP searches of RAP-DB (http://rapdb.dna.affrc.go. jp/) and MSU-RGAP (http://rice.plantbiology.msu.edu/) using the protein sequence of $q L T G 3-1$ as a query. The HyP/GRP gene family in monocots was searched by BlastP programs. The protein sequences in maize, sorghum, and Brachypodium were retrieved from Phytozome version 10 (http://phytozome.jgi.doe.gov/pz/ portal.html). Barley gene sequences were retrieved from the IPT Barley blast server (http://webblast.ipk-gatersleben.de/barley/). The $1 \mathrm{~kb}$ upstream region from the transcription start site on each gene was also retrieved from databases as the promoter sequence.

Multiple sequence alignment of the HyP/GRP protein sequences was performed using the ClustalW method with MEGA version 6 built in with a default setting (http://www. megasoftware.net/). A phylogenic tree was constructed by the neighbor-joining method considering 1000 replications with bootstrap analyses. The similarity of the amino acid sequences and identities of the promoter sequences among the HyP/GRP gene family were calculated using alignment data by the SIAS program with a default setting (http://imed.med.ucm.es/Tools/sias. $\mathrm{html}$ ). The cis-acting elements in the promoter region were analyzed using the MEME suite (http://meme.nbcr.net/meme/) with the following parameters. The optimum width of each motif was between 6 and $20 \mathrm{bp}$. The number of differential motifs was 20, while that of the minimum motif site was $5 . E=1.1 \mathrm{e}^{+4}$ was used as a threshold for shuffle sequences.

\section{COMPARATIVE ANALYSIS OF GENOME STRUCTURES AROUND qLTG3-1 ORTHOLOGOUS GENES}

Syntenic dotplots for the chromosomal regions around qLTG3-1 orthologous genes were generated using PipMaker (http:// pipmaker.bx.psu.edu/pipmaker/). The orthologous genomic regions were identified through comparative genomics analysis of the putative highly conserved gene pairs in monocots. The $163 \mathrm{~kb}$ in rice chromosome 3 including 22 genes, the $117 \mathrm{~kb}$ in Brachypodium chromosome 1 including 21 genes, the $200 \mathrm{~kb}$ in sorghum chromosome 1 including 15 genes, the $383 \mathrm{~kb}$ in maize chromosome 1 including 17 genes, and the $209 \mathrm{~kb}$ in barley chromosome 4 including 12 genes were used for the initial analysis. The CDSs in these orthologous genomic regions were then used to analyze collinearity among the monocots.

\section{DNA ANALYSIS}

Total DNA was isolated from young leaves using the CTAB method (Murray and Thompson, 1980). PCR, electrophoresis, and sequencing were performed as described previously (Fujino et al., 2004, 2005, 2010). The 1128-bp Os10g0554800 region was sequenced, including the 364 -bp $5^{\prime}$ upstream region, 504-bp coding region, and 260-bp $3^{\prime}$ downstream region. PCR products were sequenced directly using cycle sequencing with BigDye terminators (Applied Biosystems) on a Prism 3700 automated sequencer (Applied Biosystems). The sequences of four Os10g0554800 alleles were deposited in GenBank as Accession Nos. AB973302-AB973304.

\section{RNA ANALYSIS}

RNA extraction and semi-quantitative RT-PCR analysis were performed as described previously (Fujino et al., 2008a,b; Fujino and Matsuda, 2010). Total RNA was extracted from 10 embryos 
of seeds during seed germination because qLTG3-1 was specifically expressed in the embryos of germinating seeds (Fujino et al., 2008b). In addition, total RNA from the roots of 4-dayold seedlings and the 3rd leaf blades of 3-week-old seedlings were used. In semi-quantitative RT-PCR, each PCR reaction $(10 \mu \mathrm{l})$ contained $1 \mu \mathrm{l}$ of a five-fold-diluted cDNA template. The gene-specific primer sets and PCR conditions are listed in Supplemental Table S1. PCR was performed under the same conditions as those for RT-PCR using RNA without reverse transcription to determine contamination with genomic DNA. To validate the results obtained, each PCR experiment was repeated three times.

\section{RESULTS AND DISCUSSION HyP/GRP GENE FAMILY IN RICE}

To identify members of the HyP/GRP gene family in rice, the reference Nipponbare genome sequence was searched using the qLTG3-1 proteins as queries. A total of 21 genes were identified as putative HyP/GRP genes (Table 1). Amino acid alignments ranged between 124 and 184 amino acids. We renamed them OsHyPRP01 to OsHyPRP21 based on their order on the chromosome. OsHyPRP05 was qLTG3-1, which controlled lowtemperature tolerance at the seed germination stage (Fujino et al., 2004, 2008b). OsHyPRP01 and OsHyPRP13 were RCc3 and $R C c 2$, respectively, which were root-specific proteins ( $\mathrm{Xu}$ et al., 1995). The amino acid alignments of OsHyPRP revealed three conserved regions: an N-terminal region (region $\mathrm{A}$ ), variable
$\mathrm{P} / \mathrm{GRD}$ region (region $\mathrm{B}$ ), and conserved $8 \mathrm{CM}$ region (region $\mathrm{C}$ ) (Figure 1). All genes, except for OsHyPRP21, were clustered in five regions spanning 9573-38,960 bp intervals on four chromosomes. OsHyPRP21 was located on chromosome 10 apart from the $89,260 \mathrm{bp}$ of OsHyPRP20. Clusters derived by tandem duplication on chromosomes 2 and 4 and chromosomes 3 and 10 were paralogous chromosomal regions. These two pairs were previously shown to be involved in 10 major chromosometo-chromosome duplication relationships in the rice genome (Throude et al., 2009).

The OsHyPRP genes showed high similarity to each other at amino acid alignments, 0.442-0.985 (Supplemental Table S2). The highest and lowest similarities occurred between OsHyPRP06 and OsHyPRP07 and between OsHyPRP05 and OsHyPRP19, respectively. All, except for OsHyPRP05 and OsHyPRP21, showed higher similarity, a mean of 0.730 ranging 0.641-0.985. These two OsHyPRP genes, OsHyPRP05 and OsHyPRP21, had GRD and showed lower similarity with other members of the OsHyPRP genes, 0.498 and 0.539, respectively.

The phylogenic tree of the OsHyPRP genes corresponded to tandem duplications and chromosome duplications (Figure 2). The differentiation of OsHyPRPs was mainly caused by amino acid substitutions within regions $\mathrm{A}$ and $\mathrm{B}$. In contrast to the high similarity in region $\mathrm{C}, 0.920$, those in regions $\mathrm{A}$ and $\mathrm{B}$ were 0.783 and 0.586 , respectively. $\mathrm{P} / \mathrm{GRD}$ s characterized each member of the OsHyP/GRP gene family.

Table 1 | The HyP/GRP gene family in rice.

\begin{tabular}{|c|c|c|c|c|c|c|}
\hline \multirow[t]{2}{*}{ Gene name } & \multirow[t]{2}{*}{ RAP-DB } & \multirow[t]{2}{*}{ MSU_RGAP7 } & \multirow[t]{2}{*}{ Chromosome } & \multirow[t]{2}{*}{ Position } & \multicolumn{2}{|c|}{ Size } \\
\hline & & & & & $\operatorname{CDS}(b p)$ & Protein(aa) \\
\hline OsHyPRP01/RCc3 & Os02g0662000 & LOC Os02g44310 & 2 & $26,804,988-26,805,820$ & 402 & 133 \\
\hline OsHyPRPO2 & Os02g0662100 & LOC_Os02g44320 & 2 & $26,814,637-26,815,393$ & 387 & 128 \\
\hline OsHyPRPO3 & Os03g0103100 & LOC_Os03g01300 & 3 & $208,340-209,118$ & 417 & 138 \\
\hline OsHyPRPO4 & Os03g0103200 & LOC_Os03g01300 & 3 & $211,082-211,817$ & 426 & 141 \\
\hline OsHyPRP05/qLTG3-1 & Os03g0103300 & LOC_Os03g01320 & 3 & $219,977-221,070$ & 555 & 184 \\
\hline OsHyPRPO6 & Os04g0554500 & LOC_Os04g46810 & 4 & $27,740,0640-27,740,809$ & 393 & 130 \\
\hline OsHyPRPO7 & Os04g0554600 & LOC_Os04g46820 & 4 & $27,743,339-27,743,963$ & 396 & 131 \\
\hline OsHyPRPO8 & Os04g0554800 & LOC_Os04g46830 & 10 & $27,753,369-27,754,530$ & 414 & 137 \\
\hline OsHyPRPO9 & Os10g0349300 & LOC_Os10g20830 & 10 & $10,563,123-10,563,841$ & 414 & 137 \\
\hline OsHyPRP10 & Os10g0349400 & LOC_Os10g20840 & 10 & $10,566,488-10,566,901$ & 414 & 137 \\
\hline OsHyPRP11 & Os10g0349600 & LOC_Os10g20860 & 10 & $10,570,876-10,573,989$ & 402 & 133 \\
\hline OsHyPRP12 & Os10g0349900 & LOC_Os10g20890 & 10 & $10,587,298-10,587,959$ & 381 & 126 \\
\hline OsHyPRP13/RCc2 & Os10g0551800 & LOC_Os10g40430 & 10 & $21,658,192-21,658,818$ & 420 & 146 \\
\hline OsHyPRP14 & Os10g0551900 & LOC_Os10g40440 & 10 & $21,662,454-21,663,219$ & 429 & 142 \\
\hline OsHyPRP15 & - & LOC_Os10g40460 & 10 & $21,667,261-21,667,939$ & 396 & 131 \\
\hline OsHyPRP16 & Os10g0552200 & LOC_Os10g40470 & 10 & $21,670,510-21,671,244$ & 396 & 131 \\
\hline OsHyPRP17 & Os10g0552300 & LOC_Os10g40480 & 10 & $21,675,375-21,674,640$ & 411 & 136 \\
\hline OsHyPRP18 & Os10g0552600 & LOC_Os10g40510 & 10 & $21,690,332-21,691,088$ & 402 & 133 \\
\hline OsHyPRP19 & Os10g0552700 & LOC_Os10g40520 & 10 & $21,693,876-21,694,502$ & 375 & 124 \\
\hline OsHyPRP2O & Os10g0552800 & LOC_Os10g40530 & 10 & $21,697,077-21,697,778$ & 399 & 132 \\
\hline OsHyPRP21 & Os10g055480 & LOC_Os10g40614 & 10 & $21,786,108-21,787,038$ & 504 & 167 \\
\hline
\end{tabular}

RCc3, RCc2, Xu et al. (1995).

qLTG3-1; Fujino et al. (2008b). 
OsHyPRP01

OsHyPRP02

OsHyPRP03

OsHyPRP04

OsHyPRP05/qLTG3-1

OsHyPRP06

OsHyPRP07

OsHyPRP08

OsHyPRP09

OsHyPRP10

OsHyPRP11

OsHyPRP12

OsHyPRP13

OsHyPRP14

OsHyPRP15

OsHyPRP16

OsHyPRP17

OsHyPRP18

OsHyPRP19

OsHyPRP20

OsHyPRP21

OsHyPRP01

OsHyPRP02

OsHyPRP03

OsHyPRP04

OsHyPRP05/qLTG3-1

OsHyPRP06

OsHyPRP07

OsHyPRP08

OsHyPRP09

OsHyPRP10

OsHyPRP11

OsHyPRP12

OsHyPRP13

OsHyPRP14

OsHyPRP15

OsHyPRP16

OsHyPRP17

OsHyPRP18

OsHyPRP19

OsHyPRP20

OsHyPRP21

OsHyPRP01

OsHyPRP02

OsHyPRP03

OsHyPRP04

OsHyPRP05/qLTG3-1

OsHyPRP06

OsHyPRP07

OsHyPRP08

OsHyPRP09

OsHyPRP10

OsHyPRP11

OsHyPRP12

OsHyPRP13

OsHyPRP14

OsHyPRP15

OsHyPRP16

OsHyPRP17

OsHyPRP18

OsHyPRP19

OsHyPRP20

OsHyPRP21

M - . - ASKAFALFLAVNLVVLGVASA CGgSPSCPTPTPSTPTPS . . . . . . . . . . . . . .

M - - - AS - RAFLLVALNLVLFFTVASACGKYCP - TPS TP . . . $\ldots \ldots$

M - - A GKKVQVCAL FLA L NVLFTMQMGAVVQACEPYCPTPTPPVTP $\ldots \ldots$ MGGGKNKVQVCAVFVVALNMV I SMQMGAVQACEPYCPTPTPPVTP $\ldots \ldots$

M - - A TKAGVIATLLALNLHFFTFSDACGCQCG - SCPSPGGGGGGGGGGGGGGGGRGGGGGSGGGSGGG M - . - AGKAS I ALFLAVNLVVFSLASACGG . . - RCPTPTPSTPS . . . . . . . . . . . . . . . M - . - AGKAS I ALFLAVNLVVFSLGSACGG . . - HCPTPTPPTPS . . . . . . . . . . . . . . . M - . - A A K I LVFLLA I NLLFFT TANA CGCACG - KCPTPPPPALPPPP . . . . . . . . . . . . M - . - ASKVI APFLALSLLLFAVIVQGCTPNCSG . - EQVVPTPPIAVPTPS . . . . . . . . . M - . . - AS TVVAP I LALSLLLFAV IVRGCTPNCSG . . . EQVVPTPPTAVPTPL . . . . . . . . . . . M . . . ASTVVAPFLALSLLLFAVVAHGCTPNCPG . . . EQAVPATPVAVPVQS . . . . . . . . . .

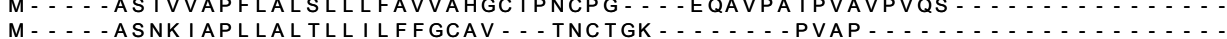
M - . - AASKVAPVLALSLLLLAVAAHGCEPHCSGGGAPAVVIPTPTVVVPLPSFGGAH .......... M - - - AYSK I APLLAL T I LLFAAAAHGCAPYCPGGAP - - PVIPTPPVVVPTPPAH - . . . . . . M - - AS TTKVVAPLLAFTLL I LAVAARGCEPNCSGG . . . . . - PVIPTP . . . . . . . . . . M - . - ASTKVVAPLLALTLLLFAVAAHGCEPNCSGGG . . . . . . . PVIPTP . . . . . . . . . . . . . M - . . - A I TKVAPLLALSLLLFAVAAVHGCEPYCGHGG - . PVIPTPPVVVP . . . . . . . . . . . . M - . - . AAKAALVLAVSLLVVAVASACTYCPEPPTPK . . . - PKPPAPR . . . . . . . . . . M $\ldots-\ldots$ A KA KALVLAVSLLAVAVAASACGTDCPP $\ldots \ldots$

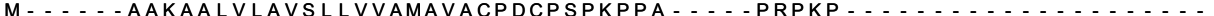
M - ASSRASASCALFLALNLLLFA I T TAC - . . . - PSCGSGGGGGHGHYGGGGSSGGGGGYGGGSGGYG

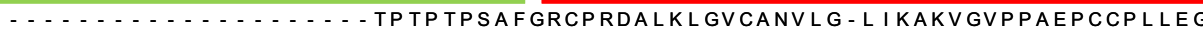

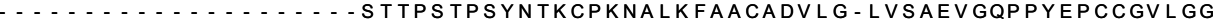

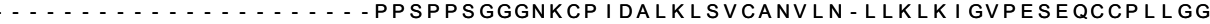

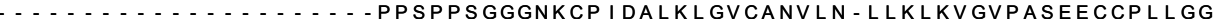
GSGGGGSGGGGSGGGGSGGGGGGGS GGGGGGGRCP I DTLKLGVCANVLNGL I NVQLGTPPRQPCCSL I QG ... . . . . . . . . . . TP TP TPAA F GK CPRDA LKL GVCANVLG - L I KAKVGVPPAEPCCPLLEG

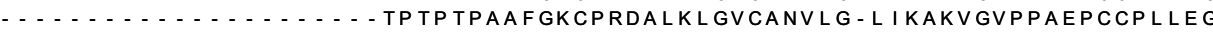

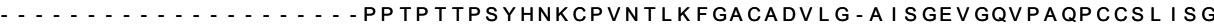

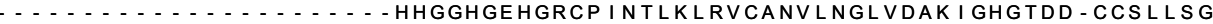

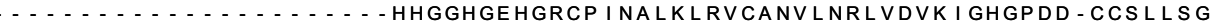

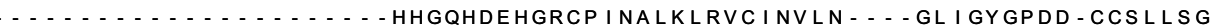
. . . . . . . . . . . . . . . . . .

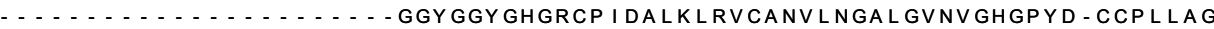

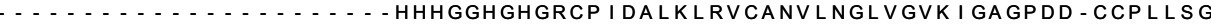

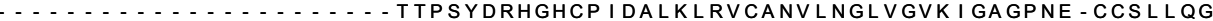
- TTPSYDRHGHCP IDALKLRVCANVLNGLVGVK IGAGPNE - CCSLLQG

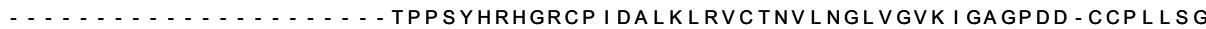

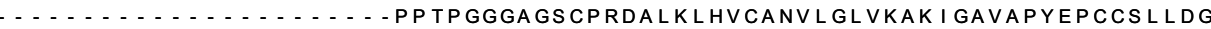

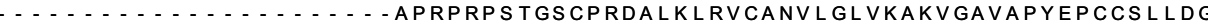

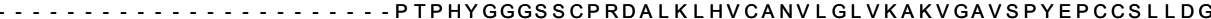
GGGS - . . . . . S SGGGYGGGGGS S TS GWYGKCP TDALKLGVCANVLD - L I KAKAGVPATEPCCPLLNG

LVDLEAAVCLCTA IRGN I LG INL - NLP I DLSL I LNYCGKTVPTGFKC - LADLEAAVCLCTA IKANVLGITL - D I PVKLSLLVNYCGKNVPSGFICA - LVDLDAAVCLCTA IKAN I LGINL - N IPVDLSLLLNYCHKTCPSDFTCPL LVDLDAAVCLCTA IKANVLG IN I - NVPVDLVLLLNYCHKTCPSDFSCPL I LADLEAAVCLCTALRAN ILGINL - NLP INLSLLVNYCGRSVPSGFQCSN LVDLEAAVCLCTA IRGN I LGINL - NLPVDLSL I LNYCGKRVPTGFKC - LVDLEAAVCLCTA IKGN I LG INL - NLPVDLSL I LNYCGKRVPTGFKCF - LADLEAAVCLCTA IKANVLGVVV - N I PVKLSLLVNYCGKCVPSGYTCA - I ADLDAAVCLCTAVKANVLG IRV - NLPVDLS IMLNKCGKTCPSDFTC - I ADLDAAVCLCTAVKANVLGIRV - NLPVDLSL I LNKCGKSCPSDFTC - IADLDAA I CLCTAVKANVLGIRV - NLPVDLGL I LNKCGKTYPTDFTC ... I ADLDAAVCLCTALKANVLGL INLNLPVDLS I I LNKCGKNYPSGFTC - . LADADAAVCLCTAVKANVLGVNL - NVPVELKL I LNKCGKTCPSDFTC - - LADLDAAVCLCTA I KANVLG I INLN I PVDLSL I LNNCGK I CPSDFTC - I ADLDAAVCLCTAVKANVLG I NL - NLPVDLSLI LNKCNK I YPSGFTC - I ADLDAAVCLCTAVKANVLGINL - NLPVDLSLILNKCSKIYPSGFTC - LADLDAAVCLCTAVKANVLGMKL - NLAVDLSL I LNKCGK I CPSDFTC - LVDLDAAVCLCTA IKANVLGLNL - N I P I DLSL I LNNCGK I CPSDYQCA - LVDLDAAVCLCTAVKANVLGIKL - DLPVDLSLI LNNCGK I CPSDFKCVH LVDLDAAVCLCTA IKANVLGIKL - NLP I DLSLILNNCGK ICPSDYQCVH LVDLEAAVCLCTA IKANVLGINL - NLPIHLSL I LNFCGKGVPTGFMCS - -

FIGURE 1 | Sequence alignment of the HyP/GRP gene family in rice. Black, green, and red lines show three conserved regions: an N-terminal region (region $\mathrm{A}$ ), variable $\mathrm{P} / \mathrm{GRD}$ region (region $\mathrm{B}$ ), and conserved $8 \mathrm{CM}$ region (region $\mathrm{C}$ ), respectively.

\section{EXPRESSION PROFILES OF THE HyP/GRP GENE FAMILY IN RICE}

To determine the expression specificity of the HyP/GRP gene family in rice, semi-quantitative RT-PCR analysis was conducted using the RNA of Hokkaiwase extracted from various tissues: embryos during seed germination at different times, the tips and bases of the roots of 4-day-old seedlings, and the 3rd leaf blades of 3-week-old seedlings (Figure 2). Seventeen of the 21 members were expressed in the different tissues of Hokkaiwase. The expression of four genes, OsHyPRP08, 11, 12, and 18, was negligible in these tissues. Gene expression levels varied among different tissues and times. OsHyPRP01 showed a similar expression profile to OsHyPRP03, specifically to that in the root. OsHyPRP06 showed a similar expression profile to OsHyPRP07, 13, 19, and 20 , specifically in the tip of the root. The gene expression of OsHyPRP05 was detected after 12-h and 2-day incubations at 30 and $15^{\circ} \mathrm{C}$ during seed germination. The expression of eight genes, OsHyPRP04, 09, 10, 14, 15, 16, 17, and 21, was detected during seed germination. These were located on chromosomes 3 and 


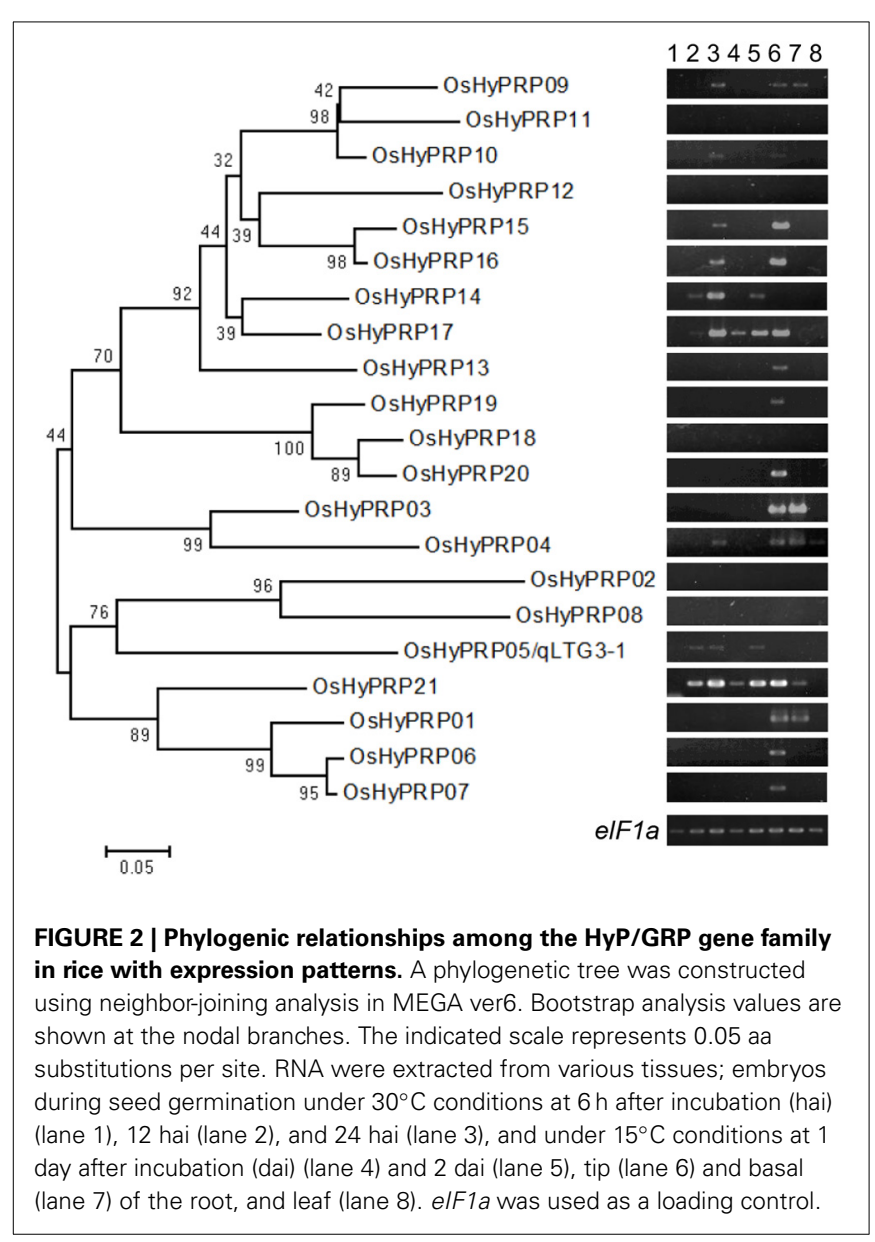

10, suggesting that paralogous genes have similar gene expression patterns. The gene expression of $O s H y P R P 21$ was higher and earlier than that of OsHyPRP05. The overlapped and different gene expression patterns of the OsHyPRP genes suggested that OsHyPRP has redundant and different roles at the developmental stages in rice.

In contrast to the high similarity of amino acid alignments, sequence identity in $1 \mathrm{~kb}$ of the $5^{\prime}$ upstream regions from UTR was low, with a mean of 0.330 ranging $0.262-0.410$ (Supplemental Table S3). Due to this low identity in the $5^{\prime}$ upstream regions, similar expression patterns may be controlled by a small number of cis-regulatory elements.

Similar expression patterns, but lower expression levels were detected in Kitaake and Hoshinoyume (Supplemental Figure S1). Since these varieties have different qLTG3-1 alleles, they exhibited different growth stages from the start of the incubation. The results strongly suggested that the expression of these OsHyPRP genes is dependent on the developmental stage based on qLTG3-1.

\section{SEQUENCE VARIATIONS IN OsHyPRP21 IN CULTIVATED RICE}

Based on chromosomal locations and the glycine-rich motif, OsHyPRP21 was considered to be a paralogous gene to OsHyPRP05. A total of 1128 nucleotides in the OsHyPRP21 (Os10g0554800) gene were sequenced among 58 varieties in WRC. Compared with the sequence of Nipponbare as a reference allele (allele A), nine mutation events at nine sites, including deletions and substitutions, were detected (Supplemental Figure S2). Only three mutation events were detected in the coding region; two deletions at positions +95 and +193 and a single nonsynonymous substitution at position +139 . These deletions occurred in-frame in the GRD, which contained Gly repeats with a Ser residue. As a result of the mutation events of these deletions, the repeat number varied. The A-G substitution at position +139 generated the amino acid substitution, Ser to Gly.

Based on these mutations, 4 different alleles were detected among WRC (Supplemental Figure S2, Table S4). Allele B, which was found in a single variety, was generated from intragenic recombinations between alleles $\mathrm{A}$ and $\mathrm{C}$. Allele $\mathrm{A}$ included 30 varieties, while alleles $C$ and $D$ included 17 and 10 varieties, respectively. A clear relationship was observed between the allele types of $O s H y P R P 21$ and the cultivar group classification. Varieties of japonica and aus had allele A, while varieties of indica had alleles $C$ and D.

Similar to qLTG3-1 (OsHyPRP05) (Fujino and Sekiguchi, 2011), the almost completely conserved protein alignment of OsHyPRP21 was identified. These results suggested that the function of OsHyPRP21 is critical at least for seed germination, during which gene expression was detected.

\section{DIVERSITY OF THE HYP/GRP GENE FAMILY IN MONOCOTS}

The sequences of four genome-sequenced species, barley, maize, Brachypodium, and sorghum, were analyzed to determine the evolutional relationships among the HyP/GRP gene family in monocots. The results of a BLAST search with qLTG3-1 as the query identified 12 genes in barley, 20 genes in maize, 10 genes in Brachypodium, and 28 genes in sorghum (Supplemental Figure S3, Table S4). All these species contained the HyP/GRP gene family, with predicted amino acid alignments ranging from 120 aa in barley to 325 aa in sorghum. Two HyP/GRP genes with a glycine rich motif was detected in barley, maize, and Brachypodium, while five were identified in sorghum. Species-specific tandem duplications occurred. In maize, four members of $\mathrm{ZmHyPRP05-}$ 08 and four members of $Z m H y P R P 16-19$ were tandem duplicated within the $52 \mathrm{~kb}$ region on chromosome 1 and the $197 \mathrm{~kb}$ region on chromosome 9, respectively. In Brachypodium, four members of $B d H y P R P 02-05$ were tandem duplicated within the $16 \mathrm{~kb}$ region on chromosomes 1 . In sorghum, three members of SbHyPRP02-04, 11 members of SbHyPRP08-18, three members of SbHyPRP20-22, and four members of SbHyPRP23-26 were tandem duplicated within the $22 \mathrm{~kb}$ region on chromosome 1 , the $101 \mathrm{~kb}$ region on chromosome 4 , the $19 \mathrm{~kb}$ region on chromosome 6 , and the $52 \mathrm{~kb}$ region on chromosome 8 , respectively.

Phylogenetic analysis revealed that a total of $91 \mathrm{HyP} / \mathrm{GRPs}$ in these five monocots appeared to be divided into seven distinct groups (Figure 3). The main difference in the amino acid alignments in each group was the alignment of the proline/glycine-rich motif (Supplemental Figure S4). The nine members of OsHyPRP, which were tandem located on chromosome 10, formed a distinct group, group V, while $\mathrm{HyP} / \mathrm{GRP}$ from other groups were clustered together in each species.

In the phylogenic tree, qLTG3-1 belonged to group VI with eight orthologous genes in monocots with marked similarity 


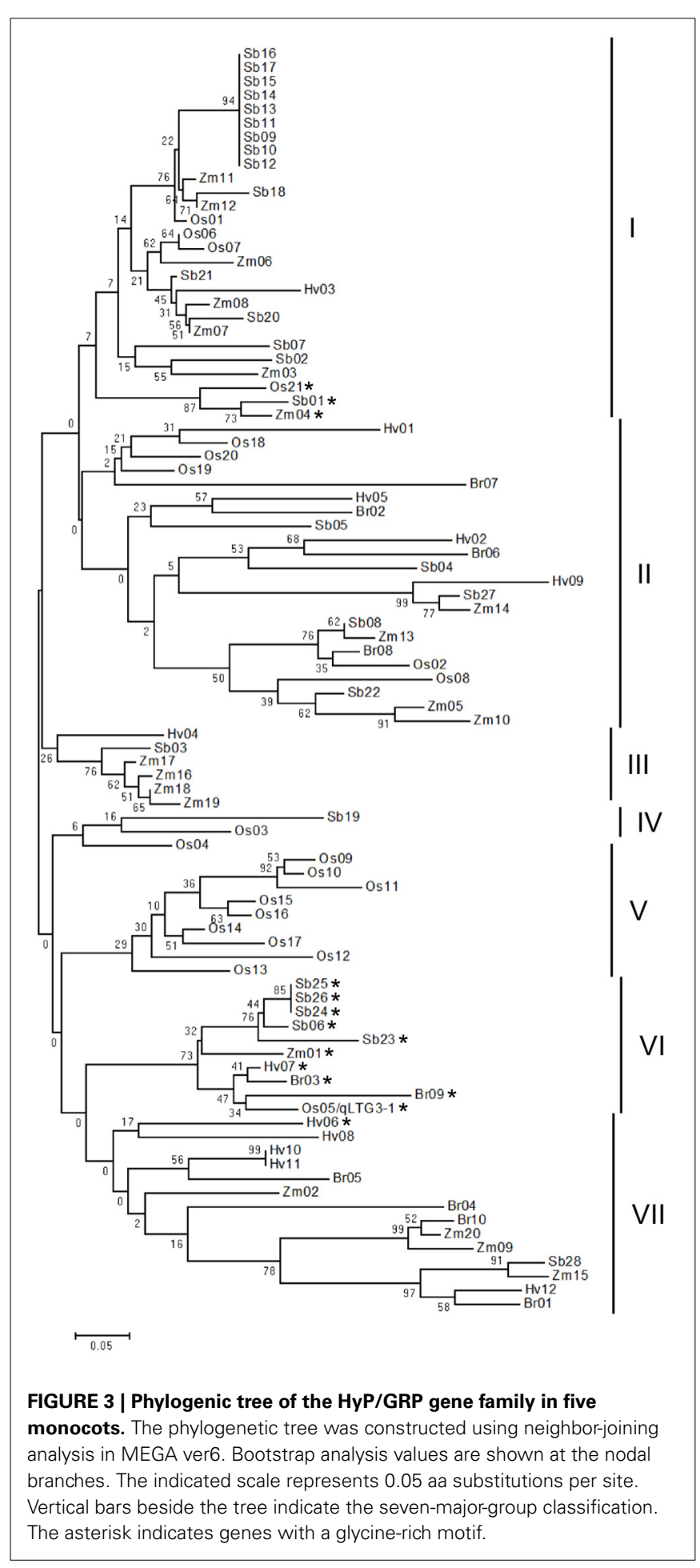

to qLTG3-1, with a mean of 0.738 and range of $0.568-1.000$ (Supplemental Table S5). All members in group VI had GRD. Three genes with GRD, OsHyPRP21, ZmHyPRP04, and $S b H y P R P 01$, formed a subcluster in group I. These results suggested that genes with a glycine-rich motif in group VI are the ancestor type, while those in group I are the paralogous type and diversified from the ancestor type. In the common ancestor of these monocots, qLTG3-1 orthologous HyP/GRP genes with GRD may be generated and at least a single duplication may have occurred.

Ten conserved amino acids, LLALNLLFFT, were previously identified at the N-termini from a comparison of qLTG3-1-like proteins in plants (Fujino et al., 2008b). An allelic variation with a single amino acid substitution, LLALNLHFFT, was then identified, which had weak function on seed germination (Hori et al., 2010; Fujino and Iwata, 2011). The LLALNLL_F_alignment was completely conserved in the nine genes for orthologous qLTG3-1 (Supplemental Figure S5), suggesting that this alignment plays a significant role in the molecular function of qLTG3-1.

In contrast to the marked similarity of amino acid alignments among the qLTG3-1 orthologous genes, sequence identity in $1 \mathrm{~kb}$ of the $5^{\prime}$ upstream regions from UTR among rice, Brachypodium, maize, and sorghum was low, with a mean of 0.422 and range of 0.274-0.756 (Supplemental Table S6). Among the three conserved motifs expected, two conserved motifs were identified as a cisregulatory motif, AGCT repeat, and ATGC repeat (Supplemental Figures S6, S7). The ATGC repeat had a CATGCA sequence, called the RY element, which was previously shown to be crucial for transactivation through $\mathrm{ABI} 3 / \mathrm{VP} 1$-like B3-domain proteins (Ezcurra et al., 2000; Reidt et al., 2000) and has been predominantly detected in seed-specific promoters (Lelievre et al., 1992). Therefore, the RY element was found in Brachypodium, maize, and sorghum, but not in rice.

Phylogenetic analysis of the HyP/GRP genes in monocots strongly suggested that the expansion of the HyP/GRP gene family occurred from the latest common ancestor of the monocots of these five species. These results indicated that orthologous HyP/GRP genes have the same function in each species. Whole genome duplications and tandem duplications after the differentiation of these species may contribute to the expansion of HyP/GRP genes as a gene family.

\section{COLLINEARITY OF CHROMOSOMAL REGIONS AROUND qLTG3-1 ORTHOLOGOUS GENES}

To clarify whether the genes in group VI originated from a common ancestral gene, we compared the chromosomal locations of these genes. OsHyPRP05/qLTG3-1 was located on rice chromosome 3. Others were BrHyPRP03 on Brachypodium chromosome 1, HvHyPRP07 on barley chromosome 4, ZmHyPRP01 on maize chromosome 1, and SbHyPRP06 on sorghum chromosome 1. A previous study reported that these chromosomes had homologous relationships (Abrouk et al., 2010). These genes among monocots were orthologous to rice $q L T G 3-1$, indicating that these genes within group VI were evolutionary related through a common ancestral gene on chromosome A7 from a putative ancestor in the proposed angiosperm evolutionary models (Abrouk et al., 2010).

Orthologous chromosomal regions were aligned and compared to identify the conservation of micro-synteny around qLTG3-1 and its orthologous genes (Supplemental Figure S8). However, only a small region was conserved among the monocots. The coding regions (CDSs) were then aligned and compared. Conserved gene alignments were detected among these species, except for barley, and high homology was detected 


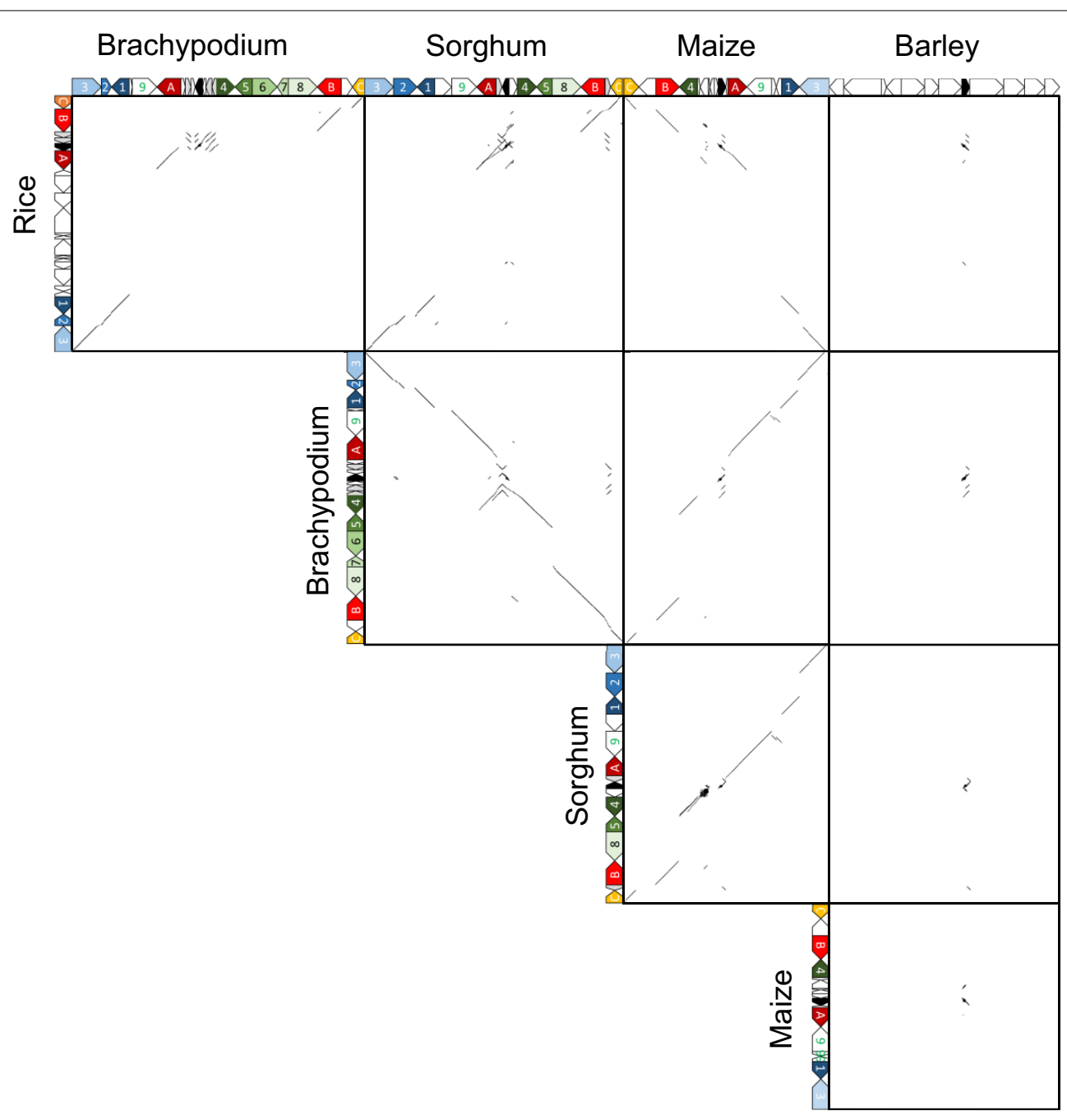

FIGURE 4 | Dot plot analysis to identify CDS regions around qLTG3-1 orthologous genes among monocots.

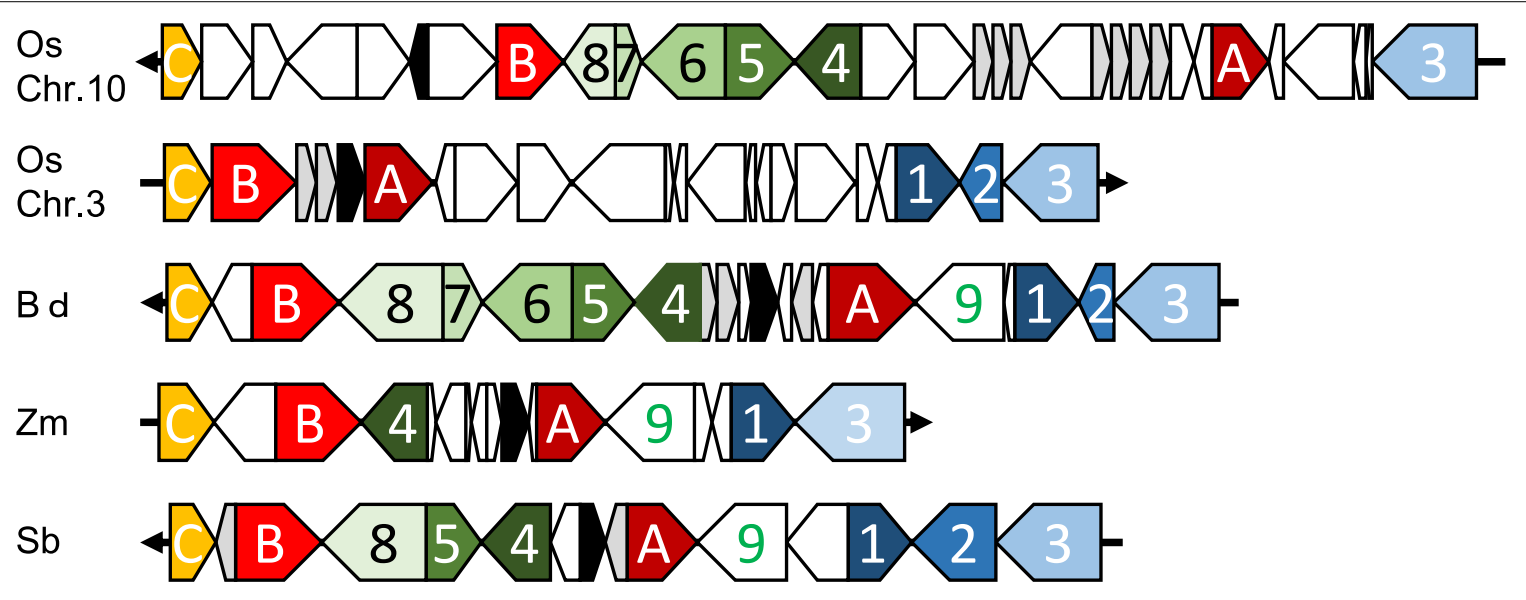

FIGURE 5 | Collinearity around qLTG3-1 orthologous genes in monocots. Os, rice; Bd, Brachypodium; Zm, maize; Sb, sorghum; Black box, qLTG3-1 orthologous gene; Gray box, HyPRP gene; White box, unique genes for each species; A, GRAS transcription factor; B, Similar to Nitrate transporter; C,
Expansin precursor; 1, Tubulin; 2, unknown protein; 3, unknown protein; 4, Exostosin-like; 5, Flavin-containing monooxygenase; 6, Unknown protein; 7, unknown protein; 8, oxysterol-binding protein; 9, Signal recognition particle receptor. 


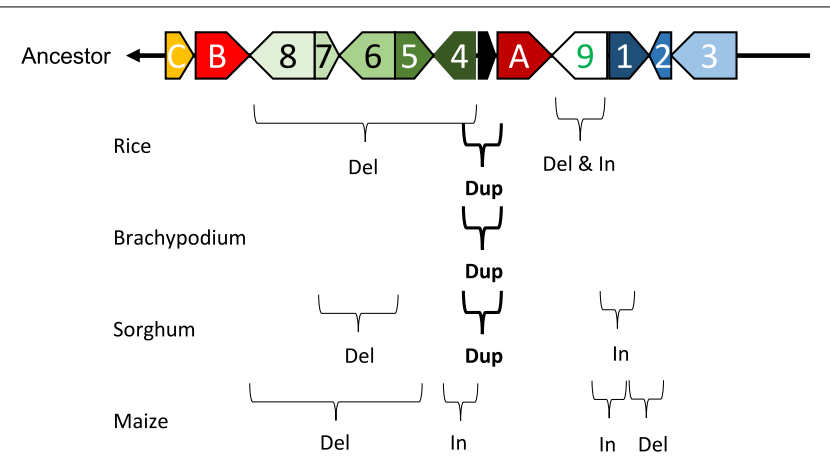

FIGURE 6 | Model of structural changes in chromosomal regions around qLTG3-1 orthologous genes during monocot diversification. Del, Dup, and In indicate deletion, duplication, and insertion, respectively. The ancestor type is modeled based on collinearity among monocots.

between homologous CDSs (Figures 4, 5). Although the number of tandem duplicated HyP/GRP genes differed, the gene order was highly conserved among maize, sorghum, and Brachypodium.

Marked genomic collinearity was observed among Brachypodium, sorghum, and maize, while collinearity could not be detected in barley. The gene order in the ancestor genome was proposed based on collinearity (Figure 6). According to the gene order, rearrangements including duplications, deletions, and insertions were involved in each monocot genome, which occurred in similar chromosomal regions.

\section{CONCLUSIONS}

HyP/GRP functions have been closely related to plant development and responses to biotic and abiotic stresses. These are involved in important agronomical traits. A clearer understanding of the molecular functions of these genes may contribute to the production of stable plants, such as low temperature germinability in rice (Fujino et al., 2004, 2008b). A comprehensive overview of the HyP/GRP gene family in rice and other monocots has been presented in this study. Based on a comparison of phylogenetic relationships, chromosomal localizations, similarity/identity, and collinearity of genomic structures around qLTG3-1 orthologous genes among monocots, the qLTG31 orthologous genes may have conserved gene function among rice, Brachypodium, maize, and sorghum.

qLTG3-1 was first identified as the genetic locus controlling low-temperature germinability in rice (Fujino et al., 2004). It was then cloned and its role was characterized in seed germination by histological observations and genome-wide expression analyses (Fujino et al., 2008b). Although the molecular function of the gene remains unclear, it may be involved in weakening the tissue covering the embryo. In barley, the coleorhiza covering the root plays a major role in causing dormancy by acting as a barrier to root emergence (Barrero et al., 2009). Comparative transcriptomics revealed that orthologous genes in syntenic genomic blocks are more likely to share correlated expression patterns (Davidson et al., 2012). The anatomical features of monocots seeds, especially the embryo and its surrounding tissue, were different. Therefore, seed germination may be controlled under the same genetic pathway including $q L T G 3-1$ and its orthologous genes.

Functional genomics in one species can be hypothesized to occur in syntenic regions in another species, namely, translational functional genomics (Paterson et al., 2010). Although rice separated from maize and sorghum $\sim 50$ million years ago (Mya) and from wheat and barley $\sim 40 \mathrm{Mya}$, their common evolutionary history can be traced by the collinear order of genetic markers across their chromosome (Rice Chromosome 3 Sequencing Consortium, 2005). In the present study, in addition to collinearity around the qLTG3-1 orthologous genes, a phylogenetic tree revealed that HyP/GRPs from different species were clustered together. A more detailed characterization of members of the OsHyPRP gene family may facilitate not only a deeper understanding of the molecular functions of HyP/GRPs, but also improved traits.

\section{AUTHOR CONTRIBUTIONS}

Conceived, designed the experiments, and wrote the manuscript: Kenji Fujino. Performed the experiments: Mari Obara, Koji Sato, Kenji Fujino. Analyzed the data: Mari Obara, Koji Sato, Kenji Fujino.

\section{ACKNOWLEDGMENTS}

This work was supported in part by grants from the Ministry of Agriculture, Forestry and Fisheries of Japan (Science and Technology Research Promotion Program for Agriculture, Forestry, Fisheries and Food Industry) and the Akiyama Life Science Foundation.

\section{SUPPLEMENTARY MATERIAL}

The Supplementary Material for this article can be found online at: http://www.frontiersin.org/journal/10.3389/fpls.2014. 00489/abstract

\section{REFERENCES}

Abrouk, M., Murat, F., Pont, C., Messing, J., Jackson, S., Faraut, T., et al. (2010) Palaeogenomics of plants: synteny-based modelling of extinct ancestors. Trends Plant Sci. 15, 479-487. doi: 10.1016/j.tplants.2010.06.001

Adams, K. L., and Wendel, J. F. (2005). Polyploidy and genome evolution in plants. Curr. Opin. Plant Biol. 8, 135-141. doi: 10.1016/j.pbi.2005.01.001

Ahn, S., and Tanksley, S. D. (1993). Comparative linkage maps of the rice and maize genomes. Proc. Natl. Acad. Sci. U.S.A. 90, 7980-7984 doi: 10.1073/pnas.90.17.7980

Barrero, J. M., Talbot, M. J., White, R. G., Jacobsen, J. V., and Gubler, F. (2009). Anatomical and transcriptomic studies of the coleorhiza reveal the importance of this tissue in regulating dormancy in barley. Plant Physiol. 150, 1006-1021. doi: 10.1104/pp.109.137901

Blanco-Portales, R., López-Raéz, J. A., Bellido, M. L., Moyano, E., Dorado, G., González-Reyes, J. A., et al. (2004). A strawberry fruit-specific and ripeningrelated gene codes for a HyPRP protein involved in polyphenol anchoring. Plant Mol Biol. 55, 763-780. doi: 10.1007/s11103-005-1966-z

Bouton, S., Viau, L., Lelièvre, E., and Limami, A. M. (2005). A gene encoding a protein with a proline-rich domain (MtPPRD1), revealed by suppressive subtractive hybridization ( $\mathrm{SSH}$ ), is specifically expressed in the Medicago truncatula embryo axis during germination. J. Exp. Bot. 56, 825-832. doi: 10.1093/jxb/eri077

Davidson, R. M., Gowda, M., Moghe, G., Lin, H., Vaillancourt, B., Shiu, S. H., et al. (2012). Comparative transcriptomics of three Poaceae species reveals patterns of gene expression evolution. Plant J. 71, 492-502. doi: 10.1111/j.1365313X.2012.05005.x 
Deutch, C. E., and Winicov, I. (1995). Post-transcriptional regulation of a saltinducible alfalfa gene encoding a putative chimeric proline-rich cell wall protein. Plant Mol. Biol. 27, 411-418. doi: 10.1007/BF00020194

Dingkuhn, M., De Datta, S. K., Pamplona, R., Javellana, C., and Schnier, H. F. (1992). Effect of late-season N-fertilization on photosynthesis and the yield of transplanted and direct-seeded tropical flooded rice. 2. A canopy stratification study. Field Crop. Res. 28, 235-249. doi: 10.1016/0378-4290(92) 90043-9

Dvoráková, L., Cvrcková, F., and Fischer, L. (2007). Analysis of the hybrid proline-rich protein families from seven plant species suggests rapid diversification of their sequences and expression patterns. BMC Genomics 8:412. doi: 10.1186/1471-2164-8-412

Ezcurra, I., Wycliffe, P., Nehlin, L., Ellerström, M., and Rask, L. (2000). Transactivation of the Brassica napus napin promoter by $\mathrm{ABI} 3$ requires interaction of the conserved B2 and B3 domains of ABI3 with different cis-elements: B2 mediates activation through an ABRE, whereas B3 interacts with an RY/G-box. Plant J. 24, 57-66. doi: 10.1046/j.1365-313x.2000.00857.x

Fujino, K., and Iwata, N. (2011). Selection for low-temperature germinability on the short arm of chromosome 3 in rice cultivars adapted to Hokkaido, Japan. Theor. Appl. Genet. 123, 1089-1097. doi: 10.1007/s00122-011-1650-4

Fujino, K., and Matsuda, Y. (2010). Genome-wide analysis of genes targeted by qLTG3-1 controlling low-temperature germinability in rice. Plant Mol. Biol. 72, 137-152. doi: 10.1007/s11103-009-9559-x

Fujino, K., Matsuda, Y., Ozawa, K., Nishimura, T., Koshiba, T., Fraaije, M. W., et al. (2008a). NARROW LEAF 7 controls leaf shape mediated by auxin in rice. Mol. Genet. Genomics 279, 499-507. doi: 10.1007/s00438-008-0328-3

Fujino, K., and Sekiguchi, H. (2011). Origins of functional nucleotide polymorphisms in a major quantitative locus, qLTG3-1, controlling low-temperature germinability in rice. Plant Mol. Biol. 75, 1-10. doi: 10.1007/s11103-0109697-1

Fujino, K., Sekiguchi, H., and Kiguchi, T. (2005). Identification of an active transposon in intact rice plants. Mol. Genet. Genomics 273, 150-157. doi: 10.1007/s00438-005-1131-z

Fujino, K., Sekiguchi, H., Matsuda, Y., Sugimoto, K., Ono, K., and Yano, M. (2008b). Molecular identification of a major quantitative trait locus, qLTG3-1, controlling low-temperature germinability in rice. Proc. Natl. Acad. Sci. U.S.A. 105, 12623-12628. doi: 10.1073/pnas.0805 303105

Fujino, K., Sekiguchi, H., Sato, T., Kiuchi, H., Nonoue, Y., Takeuchi, Y., et al. (2004). Mapping of quantitative trait loci controlling low-temperature germinability in rice (Oryza sativa L.). Theor. Appl. Genet. 108, 794-799. doi: 10.1007/s00122003-1509-4

Fujino, K., Wu, J., Sekiguchi, H., Ito, T., Izawa, T., and Matsumoto, T. (2010). Multiple introgression events surrounding the Hdl flowering-time gene in cultivated rice, Oryza sativa L. Mol. Genet. Genomics 284, 137-146. doi: 10.1007/s00438-010-0555-2

Goodwin, W., Pallas, J. A., and Jenkins, G. I. (1996). Transcripts of a gene encoding a putative cell wall-plasma membrane linker protein are specifically cold-induced in Brassica napus. Plant Mol. Biol. 31, 771-781. doi: 10.1007/BF00019465

Gu, Z., Steinmetz, L. M., Gu, X., Scharfe, C., Davis, R. W., and Li, W. H. (2003). Role of duplicate genes in genetic robustness against null mutations. Nature 421, 63-66. doi: 10.1038/nature01198

Hamilton, J. P., and Buell, C. R. (2012). Advances in plant genome sequencing. Plant J. 70, 177-190. doi: 10.1111/j.1365-313X.2012.04894.x

He, C. Y., Zhang, J. S., and Chen, S. Y. (2002). A soybean gene encoding a prolinerich protein is regulated by salicylic acid, an endogenous circadian rhythm and by various stresses. Theor. Appl. Genet. 104, 1125-1131. doi: 10.1007/s00122001-0853-5

Holk, A., Klumpp, L., and Scherer, G. F. (2002). A cell wall protein down-regulated by auxin suppresses cell expansion in Daucus carota (L.). Plant Mol. Biol. 50, 295-305. doi: 10.1023/A:1016052613196

Hori, K., Sugimoto, K., Nonoue, Y., Ono, N., Matsubara, K., Yamanouchi, U., et al. (2010). Detection of quantitative trait loci controlling preharvest sprouting resistance by using backcrossed populations of japonica rice cultivars. Theor. Appl. Genet. 120, 1547-1557. doi: 10.1007/s00122-0101275-Z

Hughes, A. L. (1994). The evolution of functionally novel proteins after gene duplication. Proc. Biol. Sci. 256, 119-124. doi: 10.1098/rspb.1994.0058
International Rice Genome Sequencing Project. (2005). The map-based sequence of the rice genome. Nature 436, 793-800. doi: 10.1038/nature03895

Josè-Estanyol, M., Ruiz-Avila, L., and Puigdomènech, P. (1992). A maize embryospecific gene encodes a proline-rich and hydrophobic protein. Plant Cell 4, 413-423. doi: 10.1105/tpc.4.4.413

Jung, H. W., Tschaplinski, T. J., Wang, L., Glazebrook, J., and Greenberg, J. T. (2009). Priming in systemic plant immunity. Science 324, 89-91. doi: 10.1126/science. 1170025

Kojima, Y., Ebana, K., Fukuoka, S., Nagamine, T., and Kawase, M. (2005). Development of an RFLP-based rice diversity research set of germplasm. Breed. Sci. 55, 431-440. doi: 10.1270/jsbbs.55.431

Lelievre, J. M., Oliveira, L. O., and Nielsen, N. C. (1992). 5'CATGCAT-3' elements modulate the expression of glycinin genes. Plant Physiol. 98, 387-391. doi: $10.1104 /$ pp.98.1.387

Lynch, M., and Force, A. (2000). The probability of duplicate gene preservation by subfunctionalization. Genetics 154, 459-473.

Murray, M. G., and Thompson, W. F. (1980). Rapid isolation of high molecular weight plant DNA. Nucleic Acids Res. 8, 4321-4325. doi: 10.1093/nar/8. 19.4321

Paterson, A. H., Bowers, J. E., Bruggmann, R., Dubchak, I., Grimwood, J., Gundlach, H., et al. (2009). The Sorghum bicolor genome and the diversification of grasses. Nature 457, 551-556. doi: 10.1038/nature07723

Paterson, A. H., Freeling, M., Haibao Tang, H., and Wang, X. (2010). Insights from the comparison of plant genome sequences. Ann. Rev. Plant Biol. 61, 349-372. doi: 10.1146/annurev-arplant-042809-112235

Peterson, M. L., Jones, D. B., and Rutger, J. N. (1978). Cool Temperature Screening of Rice Lines for Seedling Vigor. Il Riso, Vol. 27. Milan: Ente Nazionale Risi.

Priyanka, B., Sekhar, K., Reddy, V. D., and Rao, K. V. (2010). Expression of pigeonpea hybrid-proline-rich protein encoding gene (CcHyPRP) in yeast and Arabidopsis affords multiple abiotic stress tolerance. Plant Biotechnol. J. 8, 76-87. doi: 10.1111/j.1467-7652.2009.00467.x

Reidt, W., Wohlfarth, T., Ellerström, M., Czihal, A., Tewes, A., Ezcurra, I., et al. (2000). Gene regulation during late embryogenesis: the RY motif of maturationspecific gene promoters is a direct target of the FUS3 gene product. Plant J. 21, 401-408. doi: 10.1046/j.1365-313x.2000.00686.x

Rice Chromosome 3 Sequencing Consortium. (2005). Sequence, annotation, and analysis of synteny between rice chromosome 3 and diverged grass species. Genome Res. 15, 1284-1291. doi: 10.1101/gr.3869505

Scannell, D. R., Byrne, K. P., Gordon, J. L., Wong, S., and Wolfe, K. H. (2006). Multiple rounds of speciation associated with reciprocal gene loss in polyploid yeasts. Nature 440, 341-345. doi: 10.1038/nature04562

Schnable, P. S., Ware, D., Fulton, R. S., Stein, J. C., Wei, F., Pasternak, S., et al. (2009). The B73 maize genome: complexity, diversity, and dynamics. Science 326, 1112-1115. doi: 10.1126/science.1178534

Sjödin, P., Hedman, H., Kruskopf Osterberg, M., Gustafsson, S., Lagercrantz, U., and Lascoux, M. (2008). Polymorphism and divergence at three duplicate genes in Brassica nigra. J. Mol. Evol. 66, 581-590. doi: 10.1007/s00239-008-9108-2

Tan, J., Zhuo, C., and Guo, Z. (2013). Nitric oxide mediates cold- and dehydrationinduced expression of a novel MfHyPRP that confers tolerance to abiotic stress. Physiol. Plant. 149, 310-320. doi: 10.1111/ppl.12032

The International Barley Genome Sequencing Consortium. (2012). A physical, genetic and functional sequence assembly of the barley genome. Nature 491, 711-716. doi: 10.1038/nature11543

The International Brachypodium Initiative. (2010). Genome sequencing and analysis of the model grass Brachypodium distachyon. Nature 463, 763-768. doi: 10.1038/nature08747

Throude, M., Bolot, S., Bosio, M., Pont, C., Sarda, X., Quraishi, U. M., et al. (2009). Structure and expression analysis of rice paleo duplications. Nucleic Acids Res. 37, 1248-1259. doi: 10.1093/nar/gkn1048

Wang, X., Shi, X., Hao, B., Ge, S., and Luo, J. (2005). Duplication and DNA segmental loss in the rice genome: implications for diploidization. New Phytol. 165, 937-946. doi: 10.1111/j.1469-8137.2004.01293.x

Weyman, P. D., Pan, Z., Feng, Q., Gilchrist, D. G., and Bostock, R. M. (2006). DEA1, a circadian- and cold-regulated tomato gene, protects yeast cells from freezing death. Plant Mol. Biol. 62, 547-559. doi: 10.1007/s11103-006-9039-5

Wu, H. M., Zou, J., May, B., Gu, Q., and Cheung, A. Y. (1993). A tobacco gene family for flower cell wall proteins with a proline-rich domain and a cysteine-rich domain. Proc. Natl. Acad. Sci. U.S.A. 90, 6829-6833. doi: 10.1073/pnas.90.14.6829 
Xu, Y., Buchholz, W. G., DeRose, R. T., and Hall, T. C. (1995). Characterization of a rice gene family encoding root-specific proteins. Plant Mol. Biol. 27, 237-248. doi: 10.1007/BF00020180

Yeom, S. I., Seo, E., Oh, S. K., Kim, K. W., and Choi, D. (2012). A common plant cell-wall protein HyPRP1 has dual roles as a positive regulator of cell death and a negative regulator of basal defense against pathogens. Plant J. 69, 755-768. doi: 10.1111/j.1365-313X.2011.04828.x

Zhang, Y., and Schläppi, M. (2007). Cold responsive EARLI1 type HyPRPs improve freezing survival of yeast cells and form higher order complexes in plants. Planta 227, 233-243. doi: 10.1007/s00425-007-0611-2

Conflict of Interest Statement: The authors declare that the research was conducted in the absence of any commercial or financial relationships that could be construed as a potential conflict of interest.
Received: 10 July 2014; accepted: 03 September 2014; published online: 24 September 2014.

Citation: Fujino K, Obara M and Sato K (2014) Diversification of the plant-specific hybrid glycine-rich protein (HyGRP) genes in cereals. Front. Plant Sci. 5:489. doi: 10.3389/fpls.2014.00489

This article was submitted to Plant Physiology, a section of the journal Frontiers in Plant Science.

Copyright (C) 2014 Fujino, Obara and Sato. This is an open-access article distributed under the terms of the Creative Commons Attribution License (CC BY). The use, distribution or reproduction in other forums is permitted, provided the original author(s) or licensor are credited and that the original publication in this journal is cited, in accordance with accepted academic practice. No use, distribution or reproduction is permitted which does not comply with these terms. 